\title{
Fractional Chern insulators in magic-angle twisted bilayer graphene
}

https://doi.org/10.1038/s41586-021-04002-3

Received: 28 May 2021

Accepted: 7 September 2021

Published online: 15 December 2021

\section{Open access}

Check for updates

\author{
Yonglong Xie ${ }^{1,2,5 凶}$, Andrew T. Pierce ${ }^{1,5}$, Jeong Min Park ${ }^{2,5}$, Daniel E. Parker', Eslam Khalaf', \\ Patrick Ledwith', Yuan Cao' ${ }^{2}$, Seung Hwan Lee', Shaowen Chen', Patrick R. Forrester', \\ Kenji Watanabe ${ }^{3}$, Takashi Taniguchi ${ }^{4}$, Ashvin Vishwanath ${ }^{1}$, Pablo Jarillo-Herrero ${ }^{2 \bowtie} \&$ \\ Amir Yacoby ${ }^{1 凶}$
}

Fractional Chern insulators (FCls) are lattice analogues of fractional quantum Hall states that may provide a new avenue towards manipulating non-Abelian excitations. Early theoretical studie ${ }^{1-7}$ have predicted their existence in systems with flat Chern bands and highlighted the critical role of a particular quantum geometry. However, $\mathrm{FCl}$ states have been observed only in Bernal-stacked bilayer graphene (BLG) aligned with hexagonal boron nitride $(\mathrm{hBN})^{8}$, in which a very large magnetic field is responsible for the existence of the Chern bands, precluding the realization of FCIs at zero field. By contrast, magicangle twisted $\mathrm{BLG}^{9-12}$ supports flat Chern bands at zero magnetic field ${ }^{13-17}$, and therefore offers a promising route towards stabilizing zero-field FCIs. Here we report the observation of eight $\mathrm{FCl}$ states at low magnetic field in magic-angle twisted BLG enabled by high-resolution local compressibility measurements. The first of these states emerge at $5 \mathrm{~T}$, and their appearance is accompanied by the simultaneous disappearance of nearby topologically trivial charge density wave states. We demonstrate that, unlike the case of the BLG/hBN platform, the principal role of the weak magnetic field is merely to redistribute the Berry curvature of the native Chern bands and thereby realize a quantum geometry favourable for the emergence of FCls. Our findings strongly suggest that FCIs may be realized at zero magnetic field and pave the way for the exploration and manipulation of anyonic excitations in flat moiré Chern bands.
The search for novel material systems exhibiting topological properties holds promise for the next generation of electronics. For example, band-structure engineering guided by theoretical predictions has enabled the realization of integer quantized Hall states at zero magnetic field ${ }^{18-20}$, enabling new directions in spintronics and topological quantum computing. Likewise, extensive efforts have been directed towards engineering FCls-lattice analogues of fractional quantum Hall (FQH) states-in part because of their potential to manifest high-temperature topological order and to host non-Abelian excitations at zero magnetic field. However, despite a large body of theoretical work ${ }^{1-7}, \mathrm{FCl}$ states have proved exceptionally difficult to stabilize experimentally, as they require not only non-dispersive Chern bands, but also a particular quantum band geometry including a flat Berry curvature distribution. To date, FCI states have been observed only in Hofstadter bands of a BLG heterostructure aligned with $\mathrm{hBN}$ at very large $(\sim 30 \mathrm{~T})$ magnetic fields ${ }^{8}$. A key disadvantage of this platform is that its band topology fundamentally originates from the presence of the magnetic field, thus precluding the realization of FCIs in the zero-field limit.

By contrast, moiré superlattices with native topological bands ${ }^{13-17}$ provide a promising avenue to search for FCIs at zero magnetic field. In particular, the recent discovery of correlated Chern insulators (ChIs) in magic-angle twisted BLG (MATBG) down to zero field confirms the presence of intrinsic flat Chern bands ${ }^{20-29}$ and thus raises the possibility of realizing FCIs in this system. Indeed, recent analytical considerations ${ }^{30}$ and numerical calculation $\mathrm{s}^{31-33}$ have predicted FCI ground states in MATBG aligned with hBN. Importantly, these works also show the close competition between FCIs and other correlated phases such as charge density waves (CDWs), and highlight the importance of Berry curvature distribution homogeneity and the quantum metric in stabilizing FCIs in MATBG. Here we report the observation of eight FCI states at fractional fillings of the Chern bands in MATBG. The first of these states appears at $5 \mathrm{~T}$ in the range $3<v<4$, where the system is well described by an isolated Chern band. We show that these FCI states result from the intrinsic band topology of MATBG and are stabilized by weak magnetic fields that create favourable quantum geometric conditions for their emergence. The FCIs observed beyond this range, where the parent Chern states possibly reacquire their multicomponent character, are more complex, probably owing to the interplay between multiple degrees of freedom, and demonstrate the potential of MATBG for exploring novel emergent topological order.

\section{Correlated phases at fractional fillings}

To search for such topological states, we perform local electronic compressibility measurements on an MATBG device aligned with the hBN 


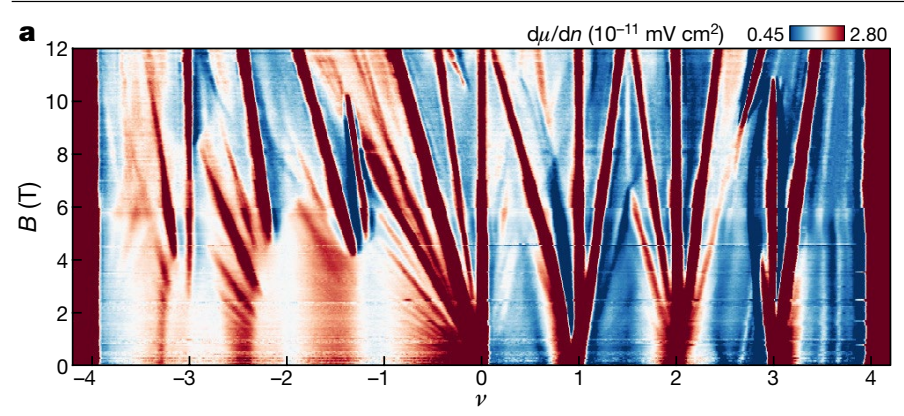

b $-\mathrm{IQH} / \mathrm{Chl}-\mathrm{Cl}-\mathrm{CDW}-\mathrm{SBCl}-\mathrm{FCl}$

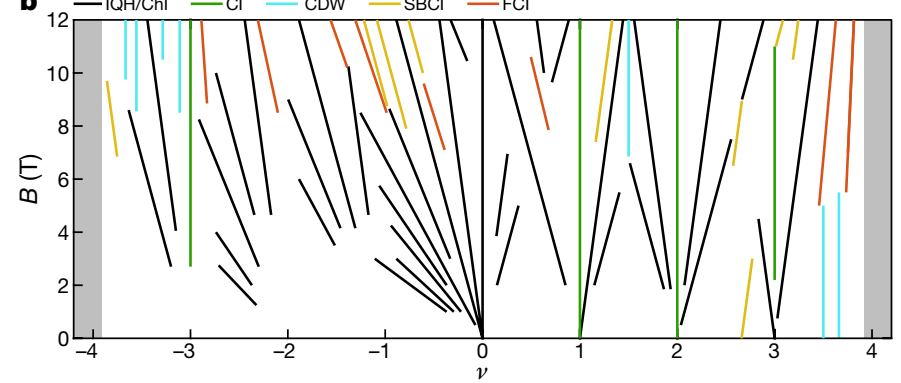

Fig. 1 Incompressible states with fractional quantum numbers in MATBG. a, Local inverse compressibility $\mathrm{d} \mu / \mathrm{d} n$ measured as a function of magnetic field $B$ and electrons per moiré unit cell $v$. b. Wannier diagram identifying the incompressible peaks present in a. Black lines correspond to ChIs and integer quantum Hall $(\mathrm{IQH})$ states; green lines correspond to correlated insulators (CIs) emanating with nonzero integer $s$ and $t=0$; blue lines correspond to CDWs with integer $t=0$ and fractional $s$; yellow lines correspond to SBCIs with nonzero integer $t$ and fractional $s$; and orange lines correspond to FCIs with fractional $t$ and fractional $s$. Grey shaded regions correspond to the gaps to the remote bands.

with a twist angle of $\sim 1.06^{\circ}$ (see Methods) using a scanning single-electron transistor (SET). Our measurements of the inverse compressibility $\mathrm{d} \mu / \mathrm{d} n$ as a function of perpendicular magnetic field $B$ and moiré band filling factor $v$ reveal a large number of linearly dispersing incompressible states (Fig. 1a, b) that can be classified by a pair of quantum numbers $(t, s)$ satisfying the Diophantine equation $v=t \phi / \phi_{0}+s$, where $v$ is the filling factor at which the incompressible peak occurs, $\phi$ is the magnetic flux per moiré unit cell, and $\phi_{0}$ is the magnetic flux quantum. We observe in total five distinct classes of incompressible states. First, incompressible features with $t=0$ and integer $s \neq 0$ correspond to trivial correlated insulators (green line in Fig. 1b). Second, features with integer $t \neq 0$ and integer $s$ correspond to integer quantum Hall states or ChIs (black lines in Fig. 1b), some of which have been identified as translation symmetry (TS)-broken states resulting from unit-cell doubling. The observed ChIs at zero field are the parent states essential for realizing more complex topological states in the zero-field limit. Finally, we observe three classes of gapped states with fractional $t$ and/or $s$, which we identify as CDWs $(t=0$ and fractional $s$ ), symmetry-broken ChIs (SBCIs-integer $t \neq 0$ and fractional $s$ ) and FCIs (fractional $t$ and fractional $s$ ).

To demonstrate that our system provides the topological bands and strong correlations essential for the realization of FCIs, we focus on the range of filling factors near $v=3$, as in this density range the band structure can be best approximated by isolated Chern bands. Figure 2a shows a measurement of inverse compressibility as a function of magnetic field for $2.5<v<4$ for $B<3 \mathrm{~T}$. In addition to the insulators emanating from $v=3$, we discover three new incompressible states that are stable down to zero magnetic field: the two non-dispersive states $(0,7 / 2)$ and $(0,11 / 3)$, which we classify as trivial CDWs, and the SBCI state (1,8/3) (Fig. 2b). The fractional values of $s$ associated with these states strongly suggest that electron-electron interactions spontaneously break the TS of the underlying moiré superlattice. In fact, a previous study ${ }^{29}$ has shown that the appearance of a portion of the ChIs is probably a consequence of TS breaking via doubling of the unit cell. In this scenario, the Hartree potential favours filling states near the centre of the mini-Brillouin zone, which in MATBG is also the region where the Berry curvature is highly concentrated (yellow trace in Fig. 2c). Consequently, the system may favour forming one band that retains the original Berry curvature and therefore has $C= \pm 1$, along

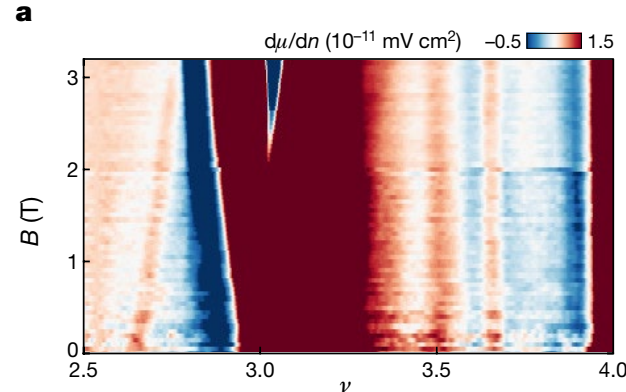

b

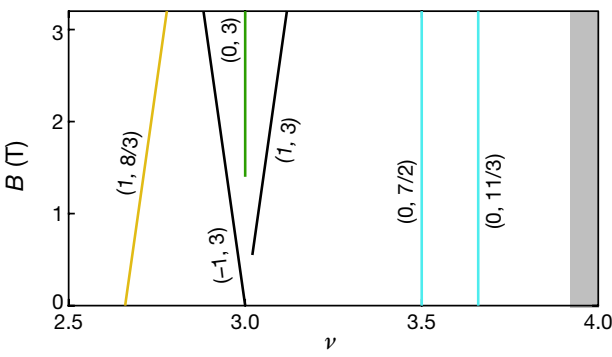

Fig. 2 Density wave states at low magnetic field for $2.5<\boldsymbol{v}<4$. a, Local inverse compressibility $\mathrm{d} \mu / \mathrm{d} n$ between $v=2.5$ and 4 for $B=0 \mathrm{~T}$ to $3 \mathrm{~T}$.b. Wannier diagram corresponding to the states observed in a coloured according to the classification used in Fig. 1b.c, Band energy (purple) and Berry curvature (yellow) along a path through the $\Gamma$ point in the first mini-Brillouin zone. Zero momentum corresponds to the $\Gamma$ point. $d$, Band structure in the case of unit-cell
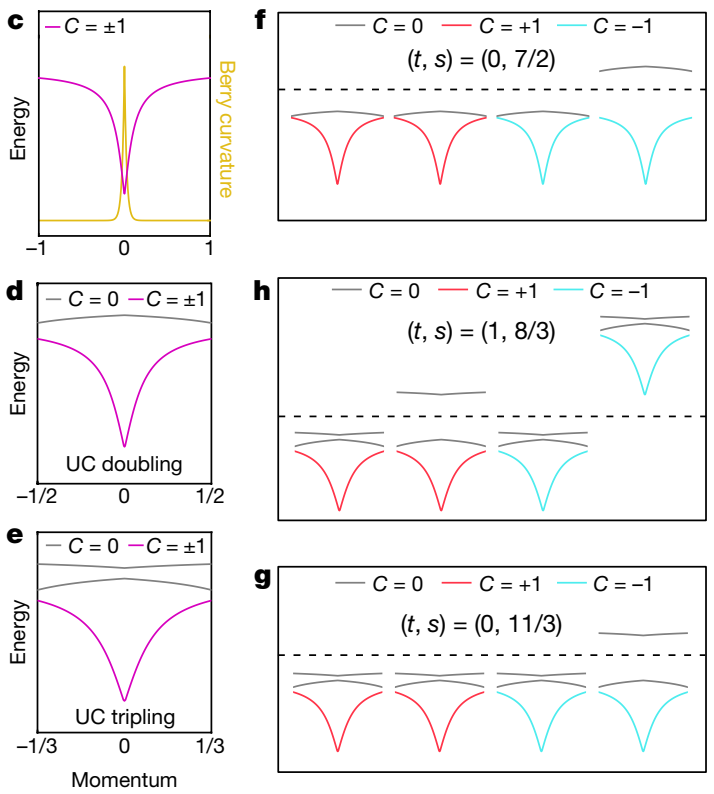

(UC) doubling resulting in a $C= \pm 1$ band accompanied by a new $C=0$ band. e, Band structure in the case of unit-cell tripling resulting in a $C= \pm 1$ band accompanied by two new $C=0$ bands. $\mathbf{f}-\mathbf{h}$, Band fillings in the case of unit-cell doubling (f) and unit-cell tripling $(\mathbf{g}, \mathbf{h})$ needed to produce the density wave states observed in $\mathbf{a}$. 

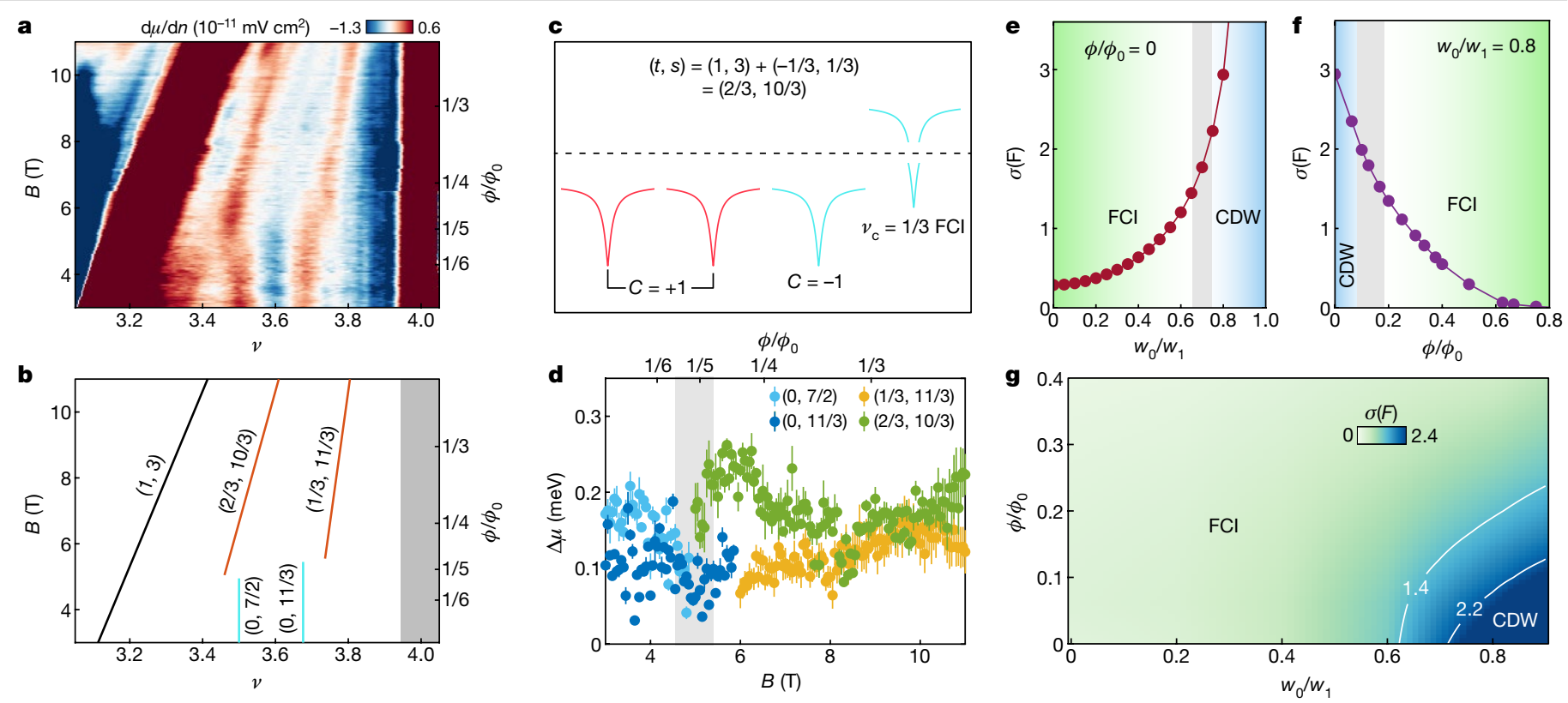

Fig. 3 | FCIs in a weak magnetic field. a, Local inverse compressibility $\mathrm{d} \mu / \mathrm{d} n$ between $v=3$ and 4 for $B=3 \mathrm{~T}$ to $11 \mathrm{~T}$. b, Wannier diagram corresponding to the states observed in a coloured according to the classification used in Fig. $1 \mathrm{~b}$. Light blue and orange lines denote the CDWs and FCIs, respectively. The grey shaded region marks the energy gap to the remote band.c, Depiction of band fillings that lead to the $(t, s)=(2 / 3,10 / 3) \mathrm{FCI}$ observed in a, which corresponds to a $v_{\mathrm{c}}=1 / 3 \mathrm{FCI}$ state from the final $C=-1$ band populated on electron-doping the $(1,3)$ ChI. d, Chemical potential steps $\Delta \mu$ associated with the CDW and FCI states observed in a obtained by integrating the inverse compressibility $\mathrm{d} \mu / \mathrm{d} n$. The error bars reflect the standard deviation obtained from fitting to $\mu(n)$.e, Calculated average Berry curvature deviation $\sigma(F)$ from the continuum model as a function of $w_{0} / w_{1}$. The grey shaded region corresponds to $w_{0} / w_{1}=0.65$ to 0.75 , the range in which the transition from FCI to CDW occurs. This $w_{0} / w_{1}$ range allows us to estimate the range of values $\sigma_{c}(F)=1.4$ to 2.2 below which the $\mathrm{FCl}$ is favourable. $f$, Calculated average Berry curvature deviation $\sigma(F)$ as a function of magnetic field for $w_{0} / w_{1}=0.8$. g, Phase diagram constructed as a function of $w_{0} / w_{1}$ and magnetic field in units of $\phi_{0}$. The white lines indicate the contours $\sigma_{\mathrm{c}}(F)=1.4$ and $\sigma_{\mathrm{c}}(F)=2.2$ that define the region where the phase boundary between the $\mathrm{FCI}$ and $\mathrm{CDW}$ ground states is expected. with a new $C=0$ band (Fig. $2 \mathrm{~d}$ ). Under this assumption, filling three of the four $C=0$ bands generated by unit-cell doubling yields the $(0,7 / 2)$ state (Fig. 2f). Similarly, tripling the unit cell allows one $C= \pm 1$ band to give rise to a $C= \pm 1$ band accompanied by two $C=0$ bands (Fig. $2 \mathrm{e}$ ). Sequentially filling the 12 reconstructed bands produces both the $(1,8 / 3)$ and $(0,11 / 3)$ states (Fig. $2 f, g)$. Together, the observation of CDW and SBCI states at zero field establishes the presence of both intrinsic band topology and strong electron-electron interactions, and highlights the critical role of the non-uniform Berry curvature in stabilizing these two classes of states.

Remarkably, on increasing the magnetic field to $5 \mathrm{~T}$, we observe a different family of robust incompressible states that are parametrized by fractional values of both $t$ and $s$ (Fig. 3a, b), characteristic of FCIs. These states, $(2 / 3,10 / 3)$ and $(1 / 3,11 / 3)$, persist up to at least $11 \mathrm{~T}$, and can be interpreted as lattice analogues of $v_{\mathrm{c}}=1 / 3$ and $2 / 3 \mathrm{FQH}$ states from the final $C=-1$ band populated on electron-doping the $(1,3) \mathrm{ChI}$, where $v_{\mathrm{c}}$ is the filling factor of the partially filled Chern band (Fig. $3 \mathrm{c}$ ). As these states do not require breaking of the TS of the moiré superlattice, they are referred to below as symmetry-preserving FCIs. These two states are expected to exhibit fractional quantized Hall conductance according to the Streda formula and hence support quasiparticle excitations with fractional charge $e / 3$ (ref. ${ }^{34}$ ), Integrating $\mathrm{d} \mu / \mathrm{d} n$ with respect to the electron density allows us to directly extract the steps in chemical potential $\Delta \mu$ associated with each of the observed CDW and FCI states (Fig. 3d). As the chemical potential is defined with respect to electrons, $\Delta \mu$ must be multiplied by the ratio of the quasiparticle charge to the electron charge, yielding energy gaps of about $50 \pm 20 \mu \mathrm{eV}$ $(\sim 0.6 \mathrm{~K})$ for both $\mathrm{FCI}$ states, roughly in agreement with the estimate of $0.01 U$ from a recent exact diagonalization study ${ }^{33}$, where $U$ is the strength of Coulomb interaction. The same study also argues that, because the spin polarization of the valley-polarized Chern band is unknown, the FCI states can be either isospin-polarized Laughlin states or multicomponent states depending on the detailed quantum geometric properties of the system. While our measurements are not capable of directly distinguishing between single and multicomponent ground states, we note that the gaps associated with both FCIs are much smaller than the spin Zeeman energy scale $E_{\mathrm{Z}}=g \mu_{\mathrm{B}} B$ (assuming $g=2)$, where $\mu_{\mathrm{B}}$ is the Bohr magneton, and depend very weakly on $B$, suggesting that the charged excitations of both states probably do not require a spin flip. The sudden appearance of the FCIs and disappearance of the CDWs indicates close competition between these two phases, with the magnetic field driving the transition yet leaving the band topology unaltered.

\section{Quantum geometry of MATBG}

To understand the transition from a CDW-dominated to an FCI-dominated regime, we begin by observing that these two classes of ground states place very different constraints on the quantum geometric properties of the underlying band structure of MATBG. For the CDW ground states to emerge, the Berry curvature of the flat bands must be strongly concentrated near the centre of the mini-Brillouin zone to take advantage of the Hartree potential ${ }^{29}$. However, bands with sufficiently nonuniform Berry curvature are known to disfavour FCI ground states ${ }^{30-33}$. The observed transition therefore suggests that the applied magnetic field in the experiment serves primarily to reduce the intrinsic Berry curvature inhomogeneity within the partially filled Chern band, unlike in the hBN/BLG system where the applied field is needed to produce Chern bands in the first place. To estimate the amount of Berry curvature inhomogeneity the FCI ground states can tolerate, we note that exact diagonalization studies ${ }^{31-33}$ indicate a transition between CDW and $\mathrm{FCI}$ ground states as a function of $w_{0} / w_{1}$, where 

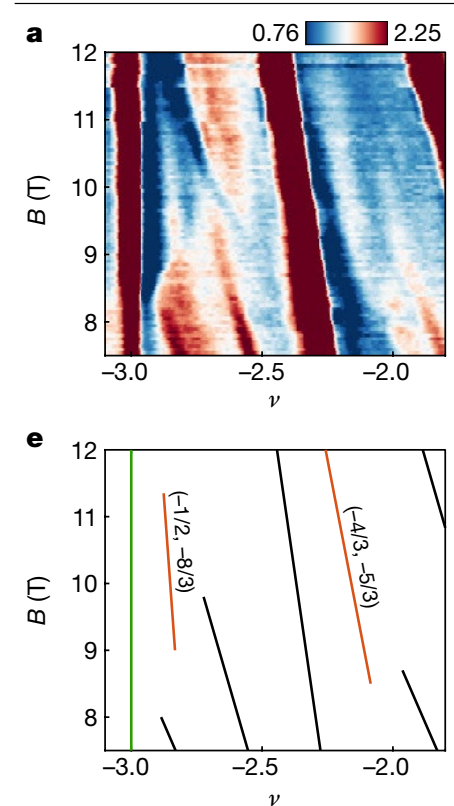

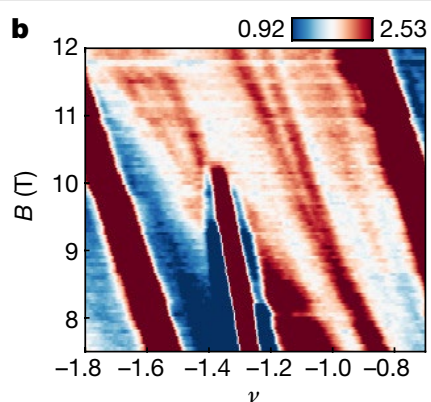

$\mathbf{f}$

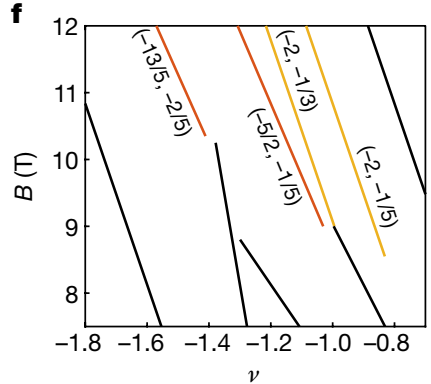

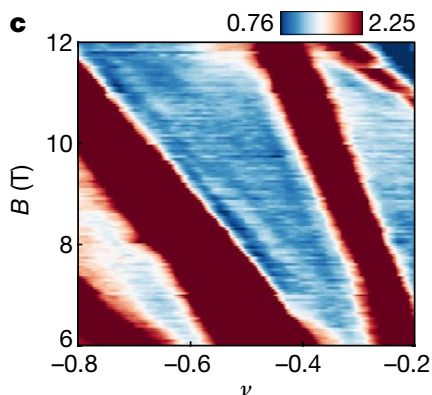

g

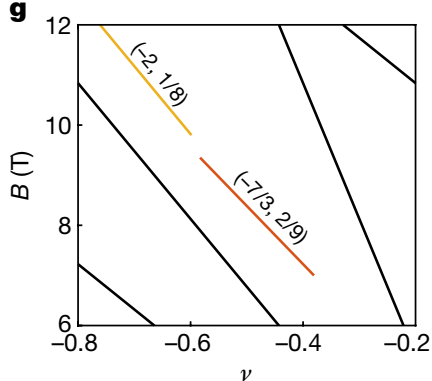

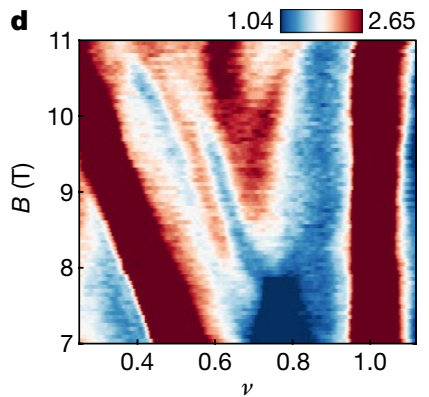

h

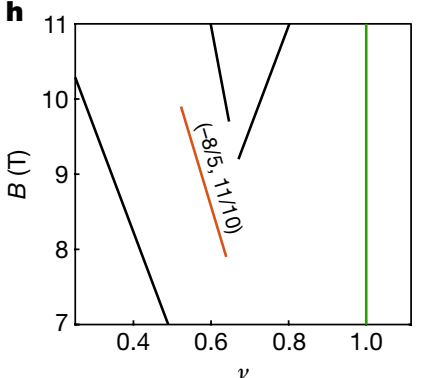

Fig. 4 | Additional FCIs at higher magnetic field. a-d, Measurements of $\mathrm{d} \mu / \mathrm{d} n\left(\times 10^{-11} \mathrm{mV} \mathrm{cm}^{-2}\right)$ in various density ranges between 6 and $12 \mathrm{~T}$ showing additional FCIs and SBCIs. $\mathbf{e}-\mathbf{h}$, Wannier diagrams corresponding to the states

$w_{0}$ and $w_{1}$ are the interlayer tunnelling matrix elements at the AA-stacked and the $A B$-stacked regions, respectively. This ratio is known to strongly alter the Berry curvature distribution within the flat bands of MATBG. According to these works, the transition occurs near $w_{0} / w_{1} \approx 0.7$, as has been confirmed by a recent density matrix renormalization group study (D.E.Parker et al., manuscript in preparation). The ground-state dependence on $w_{0} / w_{1}$ therefore gives a means of parametrizing the dependence of the FCI ground states on the Berry curvature inhomogeneity, which we characterize using the quantity $\sigma(F)$, the mean standard deviation of the Berry curvature over the mini-Brillouin zone. We estimate an upper bound on the allowable Berry curvature inhomogeneity $\sigma_{\mathrm{c}}(F)$ to be in the range of 1.4 to 2.2, depending on the model parameters, below which the quantum geometry of the system is favourable for the emergence of FCIs (Fig. 3e). For simplicity, we choose $\sigma_{\mathrm{c}}(F)=1.8$ for the discussion below. For realistic MATBG samples, $w_{0} / w_{1}$ is estimated to be around 0.8 (refs. ${ }^{25,35,36}$ ), yielding large values of $\sigma(F) \sim 3$, consistent with our observation of CDW states at zero field. Thus, the absence of $\mathrm{FCI}$ ground states at zero magnetic field in our device can be understood to result from the large values of $\sigma(F)$ present in MATBG.

Having established the critical role of $\sigma(F)$ in determining the many-body ground state, we now examine its evolution as a function of magnetic field by analysing the Hofstadter spectrum of the continuum model of MATBG aligned with hBN (see Methods). We find that increasing the magnetic field reduces $\sigma(F)$ monotonically (Fig. 3f), with $\sigma(F)$ vanishing as $\phi / \phi_{0} \rightarrow 1$. To estimate the value of magnetic field at which the $\mathrm{FCI}$ ground state becomes favourable, we identify the magnetic field, $B_{\mathrm{c}}$, at which $\sigma(F)$ falls below the critical value $\sigma_{\mathrm{c}}(F) \approx$ 1.8. For realistic values of $w_{0} / w_{1} \sim 0.8$, our calculations find that $\sigma(F)$ is reduced below $\sigma_{\mathrm{c}}$ starting at $\phi / \phi_{0} \sim 1 / 5$ or $B_{\mathrm{c}} \sim 5.4 \mathrm{~T}$, in good agreement with the magnetic field at which the $(2 / 3,10 / 3)$ and $(1 / 3,11 / 3)$ states appear experimentally. We emphasize that due to the sharp decrease of $\sigma(F)$ with field, the critical field $B_{\mathrm{c}}$ is not sensitive to the precise choice of $\sigma_{\mathrm{c}}(F)$. Combining the bound $\sigma_{\mathrm{c}}(F)$ estimated from many-body ground state analyses ${ }^{31-33}$ (D.E.P., manuscript in preparation) at $B=0$ with our calculations of $\sigma(F)$ as a function of $B$ and $w_{0} / w_{1}$ allows us to sketch a phase diagram at $v_{\mathrm{c}}=1 / 3$ (Fig. $3 \mathrm{~g}$ ). Our calculations also demonstrate that the $\mathrm{FCI}$ is adiabatically connected to the $\mathrm{FQH}$ state at

observed in a-d coloured according to the classification used in Fig. 1b. Green, yellow and orange lines denote the correlated insulators, SBCIs and FCIs, respectively. $\phi / \phi_{0}=1$, where the band geometry reduces to that of the lowest Landau level. However, unlike the case of the usual FQH states or of FCIs occurring within partially filled Hofstadter bands in a BLG/hBN heterostructure, in which the Berry curvature is supplied by the Landau levels or Chern bands that form in a magnetic field, the FCIs observed here fundamentally stem from zero-field ChI parent states, and the only role of the magnetic field is to flatten the Berry curvature. Therefore, only a weak magnetic field of less than $20 \%$ of a magnetic flux quantum per moiré unit cell is required to stabilize the FCIs by reducing $\sigma(F)$ below $\sigma_{\mathrm{c}}(F)$.

\section{FCIs away from $3<v<4$}

Outside the density range $3<v<4$, the system recovers additional degrees of freedom and thus permits more possible competing ground states at fractional fillings. In particular, we observe six additional FCIs-along with numerous SBCIs with denominators of $s$ as large as 10 (Extended Data Fig. 1) - at slightly higher values of magnetic field, particularly on the hole side (Fig. 4), most of which show values of $\Delta \mu$ comparable to those of their counterparts near $v=3$ (Extended Data Fig. 2). We emphasize that our measurements unambiguously identify these states as FCIs purely on the basis of the Streda formula regardless of their exact nature and origin, on which we speculate below. As in the case of the FCIs observed for $3<v<4$, several of these additional states probably correspond to symmetry-preserving FCIs (Fig. 4a, b). For example, we interpret the state $(-4 / 3,-5 / 3)$ as arising from a $v_{\mathrm{c}}=1 / 3 \mathrm{FCI}$ formed within the $C=-1$ band populated on electron-doping the $(-1$, $-2)$ ChI, similar to the $(2 / 3,10 / 3)$ and $(1 / 3,11 / 3)$ states described above. In addition, unlike the aforementioned states, the observed $(-8 / 5$, $11 / 10)$ and $(-7 / 3,2 / 9)$ states (Fig. $4 \mathrm{c}, \mathrm{d})$ have $s$ with denominator a multiple of that of $t$, rather than being equal, suggesting that each unit cell binds only a fraction of an electron charge and that the states therefore correspond to symmetry-broken FCIs. Specifically, the $(-8 / 5,11 / 10)$ state can result from doping the $(-2,1) \mathrm{ChI}$ with a $v_{\mathrm{c}}=2 / 5 \mathrm{FCI}$ that quadruples the unit cell and thus contributes to a change in $s$ of only $1 / 10$. This interpretation is further supported by the fact that the $(-2,1) \mathrm{ChI}$ is a state that breaks TS, and thus might naturally be expected to also support symmetry-broken FCIs. We note that such symmetry-broken 
$\mathrm{FCl}$ states have no analogue in the FQH system. Most intriguingly, we also find evidence of FCI states with coprime denominators of $t$ and $s$ that as a result cannot be described as either symmetry-preserving or symmetry-breaking FCIs. The emergence of these exotic many-body ground states may originate from complex interplay between spin, valley and spatial symmetry.

The observation of FCIs in MATBG reported here leaves open many theoretical and experimental questions. An interesting and straightforward direction is to identify the quasiparticle charge associated with these $\mathrm{FCI}$ states, especially those that have no analogues in the FQH system. The competition between FCIs and nearby CDWs and SBCIs may provide a new setting for the study of quantum phase transitions. Importantly, our work establishes the applied magnetic field as a novel tuning knob for the Berry curvature distribution, and indicates close proximity to zero-field FCIs in the flat bands of MATBG. Thus, a pressing experimental task is to develop means of reducing $w_{0} / w_{1}$ in MATBG and to explore alternative platforms beyond MATBG that suffer less from Berry curvature inhomogeneity, which would enable the realization of FCIs at zero magnetic field and offer new opportunities for the creation of next-generation topological quantum devices.

\section{Online content}

Any methods, additional references, Nature Research reporting summaries, source data, extended data, supplementary information, acknowledgements, peer review information; details of author contributions and competing interests; and statements of data and code availability are available at https://doi.org/10.1038/s41586-021-04002-3.

1. Sheng, D. N., Gu, Z.-C., Sun, K. \& Sheng, L. Fractional quantum Hall effect in the absence of Landau levels. Nat. Commun. 2, 389 (2011).

2. Neupert, T., Santos, L., Chamon, C. \& Mudry, C. Fractional quantum Hall states at zero magnetic field. Phys. Rev. Lett. 106, 236804 (2011).

3. Regnault, N. \& Bernevig, B. A. Fractional Chern insulator. Phys. Rev. X1, 021014 (2011).

4. Tang, E., Mei, J.-W. \& Wen, X.-G. High-temperature fractional quantum Hall states. Phys. Rev. Lett. 106, 236802 (2011).

5. Qi, X.-L. Generic wave-function description of fractional Quantum anomalous Hall states and fractional topological insulators. Phys. Rev. Lett. 107, 126803 (2011).

6. Parameswaran, S. A., Roy, R. \& Sondhi, S. L. Fractional quantum Hall physics in topological flat bands. C. R. Phys. 14, 816-839 (2013).

7. Bergholtz, E. J. \& Liu, Z. Topological flat band models and fractional Chern insulators. Int. J. Mod. Phys. B 27, 1330017 (2013).

8. Spanton, E. M. et al. Observation of fractional Chern insulators in a van der Waals heterostructure. Science 360, 62-66 (2018).

9. Bistritzer, R. \& MacDonald, A. H. Moiré bands in twisted double-layer graphene. Proc. Natl Acad. Sci. USA 108, 12233-12237 (2011).

10. Suárez Morell, E., Correa, J. D., Vargas, P., Pacheco, M. \& Barticevic, Z. Flat bands in slightly twisted bilayer graphene: tight-binding calculations. Phys. Rev. B 82, 121407 (2010).

11. Cao, Y. et al. Correlated insulator behaviour at half-filling in magic-angle graphene superlattices. Nature 556, 80-84 (2018).

12. Cao, Y. et al. Unconventional superconductivity in magic-angle graphene superlattices. Nature 556, 43-50 (2018)

13. Po, H. C., Zou, L., Vishwanath, A. \& Senthil, T. Origin of Mott insulating behavior and superconductivity in twisted bilayer graphene. Phys. Rev. X 8, 031089 (2018).

14. Song, Z. et al. All magic angles in twisted bilayer graphene are topological. Phys. Rev. Lett. 123, 036401 (2019).
15. Ahn, J., Park, S. \& Yang, B.-J. Failure of Nielsen-Ninomiya theorem and fragile topology in two-dimensional systems with space-time inversion symmetry: application to twisted bilayer graphene at magic angle. Phys. Rev. X 9, 021013 (2019).

16. Po, H. C., Zou, L., Senthil, T. \& Vishwanath, A. Faithful tight-binding models and fragile topology of magic-angle bilayer graphene. Phys. Rev. B 99, 195455 (2019).

17. Liu, J., Liu, J. \& Dai, X. Pseudo Landau level representation of twisted bilayer graphene: band topology and implications on the correlated insulating phase. Phys. Rev. B 99, 155415 (2019).

18. Chang, C.-Z. et al. Experimental observation of the quantum anomalous Hall effect in a magnetic topological insulator. Science 340, 167-170 (2013).

19. Deng, Y. et al. Quantum anomalous Hall effect in intrinsic magnetic topological insulator $\mathrm{MnBi}_{2} \mathrm{Te}_{4}$. Science 367, 895-900 (2020).

20. Serlin, M. et al. Intrinsic quantized anomalous Hall effect in a moiré heterostructure. Science 367, 900-903 (2020).

21. Sharpe, A. L. et al. Emergent ferromagnetism near three-quarters filling in twisted bilayer graphene. Science 365, 605-608 (2019).

22. Nuckolls, K. P. et al. Strongly correlated Chern insulators in magic-angle twisted bilayer graphene. Nature https://doi.org/10.1038/s41586-020-3028-8 (2020).

23. Wu, S., Zhang, Z., Watanabe, K., Taniguchi, T. \& Andrei, E. Y. Chern insulators, van Hove singularities and topological flat bands in magic-angle twisted bilayer graphene. Nat. Mater. 20, 488-494 (2021).

24. Saito, Y. et al. Hofstadter subband ferromagnetism and symmetry-broken Chern insulators in twisted bilayer graphene. Nat. Phys. 17, 478-481 (2021).

25. Das, I. et al. Symmetry-broken Chern insulators and Rashba-like Landau-level crossings in magic-angle bilayer graphene. Nat. Phys. https://doi.org/10.1038/s41567-021-01186-3 (2021).

26. Choi, Y. et al. Correlation-driven topological phases in magic-angle twisted bilayer graphene. Nature 589, 536-541 (2021).

27. Park, J. M., Cao, Y., Watanabe, K., Taniguchi, T. \& Jarillo-Herrero, P. Flavour Hund's coupling, Chern gaps and charge diffusivity in moiré graphene. Nature 592, 43-48 (2021).

28. Stepanov, P. et al. Competing zero-field Chern insulators in superconducting twisted bilayer graphene. Preprint at https://arxiv.org/abs/2012.15126 (2020).

29. Pierce, A. T. et al. Unconventional sequence of correlated Chern insulators in magic-angle twisted bilayer graphene. Nat. Phys. 17, 1210-1215 (2021).

30. Ledwith, P. J., Tarnopolsky, G., Khalaf, E. \& Vishwanath, A. Fractional Chern insulator states in twisted bilayer graphene: an analytical approach. Phys. Rev. Res. 2, 023237 (2020).

31. Abouelkomsan, A., Liu, Z. \& Bergholtz, E. J. Particle-hole duality, emergent Fermi liquids, and fractional Chern insulators in moiré flatbands. Phys. Rev. Lett. 124, 106803 (2020).

32. Wilhelm, P., Lang, T. C. \& Läuchli, A. M. Interplay of fractional Chern insulator and charge density wave phases in twisted bilayer graphene. Phys. Rev. B 103, 125406 (2021).

33. Repellin, C. \& Senthil, T. Chern bands of twisted bilayer graphene: fractional Chern insulators and spin phase transition. Phys. Rev. Res. 2, 023238 (2020).

34. Laughlin, R. B. Anomalous quantum Hall effect: an incompressible quantum fluid with fractionally charged excitations. Phys. Rev. Lett. 50, 1395-1398 (1983).

35. Carr, S., Fang, S., Zhu, Z. \& Kaxiras, E. Exact continuum model for low-energy electronic states of twisted bilayer graphene. Phys. Rev. Res. 1, 013001 (2019).

36. Xie, Y. et al. Spectroscopic signatures of many-body correlations in magic-angle twisted bilayer graphene. Nature $\mathbf{5 7 2}, 101-105$ (2019).

Publisher's note Springer Nature remains neutral with regard to jurisdictional claims in published maps and institutional affiliations.

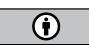

Open Access This article is licensed under a Creative Commons Attribution 4.0 International License, which permits use, sharing, adaptation, distribution and reproduction in any medium or format, as long as you give appropriate credit to the original author(s) and the source, provide a link to the Creative Commons license, and indicate if changes were made. The images or other third party material in this article are included in the article's Creative Commons license, unless indicated otherwise in a credit line to the material. If material is not included in the article's Creative Commons license and your intended use is not permitted by statutory regulation or exceeds the permitted use, you will need to obtain permission directly from the copyright holder. To view a copy of this license, visit http://creativecommons.org/licenses/by/4.0/.

(c) The Author(s) 2021 


\section{Methods}

\section{Sample preparation}

The MATBG device used in this study was fabricated using the 'tear-and-stack' technique described in refs. ${ }^{37,38}$, and is the same as the one in ref. ${ }^{29}$. Briefly, the monolayer graphene and hBN flakes were first exfoliated on $\mathrm{SiO}_{2} / \mathrm{Si}$ substrates and subsequently screened with optical microscopy and atomic force microscopy. We use a PC/PDMS stamp on a glass slide to sequentially pick up the flakes. The resulting stack is released on the pre-stacked hBN-on-Pd/Au back gate. The device geometry was defined by electron-beam lithography and reactive ion etching. $\mathrm{Cr} / \mathrm{Au}$ electrical contacts to MATBG were made by the standard edge-contact method.

\section{Compressibility measurements}

All compressibility measurements were made in $\mathrm{a}^{3} \mathrm{He}$ cryostat. The SET tips were fabricated using a procedure described elsewhere ${ }^{39}$. Compressibility measurements were performed using a.c. and d.c. protocols similar to those described in refs. ${ }^{29,39}$. a.c. excitations of $40 \mathrm{mV}$ at $97.17 \mathrm{~Hz}$ and $1.5 \mathrm{mV}$ at $107.17 \mathrm{~Hz}$ were applied to the back gate and to MATBG, respectively. The tip was held approximately $100 \mathrm{~nm}$ above the MATBG. A d.c. feedback loop was used to hold the phase of the SET's Coulomb blockade signal fixed, which results in a direct d.c. measurement of the chemical potential. Compared to ref. ${ }^{29}$, a longer integration and voltage-ramping time and a slightly smaller tip-sample distance were used to further improve the signal-to-noise ratio.

\section{Procedure of determining the slope and the intercept for the fractional states}

We determine the quantum numbers $(t, s)$ of the incompressible states by first identifying the peaks associated with each state and performing a linear fit to obtain their slope and intercept. To more accuratelyconfirm the fractional values of $t$ and $s$ and mitigate the error due to effects of quantum capacitance ${ }^{8}$, we use the fitted slope and intercept of the nearby Chern and correlated insulators to obtain local estimates of $t$ and $s$. On the basis of the converted values of $t$ and $s$, we assign the corresponding fractions for $t$ and $s$ by identifying those with the smallest denominator possible (up to 10 ) within the $95 \%$ confidence interval and favour the fractions of $t$ and $s$ that share the same denominators (Extended Data Fig. 3).

\section{TS breaking}

We briefly summarize the origin of TS breaking in MATBG and its consequence on the topological Chern structure reported in ref. ${ }^{29}$. It has been shown that the strong Hartree potential of the flat bands of MATBG favours populating states at the centre (corner) of MATBG's mini-Brillouin zone for the electron-doped (hole-doped) side (see Fig. 2c for the electron-doped case). As a result, the system can lower its energy by enlarging the moiré unit cell (doubling, tripling, quadruplingand so on), thereby breaking TS to form an insulator. The immediate consequence of enlarging the moire unit cell is to fold the original bands, which results in a new set of $N$ reconstructed bands, where $N$ is the factor by which the unit cell is enlarged. For example, doubling (tripling) the unit cell leads to two (three) reconstructed bands (Fig. 2d, e) per flavour. As the Berry curvature is highly concentrated at the centre of the mini-Brillouin zone (Fig. 2c), the lowest (highest) band retains the Chern number of the original band for the electron-doped (hole-doped) side, while the rest of the reconstructed bands carry zero Chern number. This picture captures the unconventional sequence of ChIs as well as the zero-field SBCIs and CDWs we observe in the present work.

\section{Influence of twist angle inhomogeneity on FCIs}

We have examined the twist angle inhomogeneity over a distance of approximately $1.6 \mu \mathrm{m}$, and we find the local twist angle to vary by approximately $0.005^{\circ}$ (Extended Data Fig. 4a). Extended Data Fig. 4b shows the compressibility measurements between $v=3$ and 4 taken at $9 \mathrm{~T}$, where the incompressible peaks associated with $(0,3)$ and $(1,3)$ shift in density owing to the changes in the local twist angle. Similarly, the incompressible features that appear in the density range where the $(2 / 3,10 / 3)$ and $(1 / 3,11 / 3) \mathrm{FCI}$ states are expected to occur-indicated by the blue and black arrows, respectively-also display shifts in density. To unambiguously identify the nature of these incompressible peaks, we have examined their magnetic field dependence at three different locations (Extended Data Fig. 4c). We find that, at location 2, where the variation of twist angle is small, the evolution of the two incompressible peaks indeed follows the Diophantine equation with $t=2 / 3$ and $t=1 / 3$, demonstrating the robust reproducibility of the observation of the FCIs. At location 1 , the peaks associated with $t=2 / 3$ and $t=1 / 3$ are also present over most of the field range, but the appearance of additional incompressible peaks suggests that location 1 is near a region where local disorder different from twist angle inhomogeneity is present. However, at location 3 where the twist angle varies rapidly, the left incompressible peak deviates from the expected trajectory for a $t=2 / 3$ FCI and may be better described by a $C=1 \mathrm{SBCI}$ emanating from $s=13 / 4$. Overall, these measurements demonstrate the robustness of the FCI ground states and highlight the critical role of twist angle homogeneity in stabilizing the FCIs, lending further support to the idea that controlling local microscopic parameters may provide a pathway for tuning transitions between CDWs/SBCIs and FCIs.

\section{Hofstadter spectrum}

We model the system using the Bistritzer-MacDonald model ${ }^{9}$ with a twist angle of $\theta=1.06^{\circ}$, and account for the gap at charge neutrality observed in the experiment by including a sublattice splitting of $30 \mathrm{meV}$. The interlayer tunnelling parameter $w_{1}$ is set to $110 \mathrm{meV}$. In the range $3<v<4$, the system can be approximated by a single Chern band, and we thus consider a single fermion species in our calculation. We obtain the Hofstadter spectrum following refs. ${ }^{40-42}$, which is shown in Extended Data Fig. 5. As dictated by the Streda formula, the top $C=-1$ band-the parent state of the $\mathrm{FCI}-$ is separated by a gap. Complete details of the model are given in the Supplementary Information.

\section{Quantum geometry}

The stability of $\mathrm{FCl}$ is closely related to the quantum geometry of the MATBG band structure. One key figure of merit is the standard deviation of the Berry curvature distribution over the Brillouin zone. In the presence of a magnetic flux $\phi=\frac{p}{q} \phi_{0}$, there are $2 q$ bands in the Hofstadter spectrum, where $q-C p$ bands are filled. Here we present a natural multi-band generalization for the standard deviation of the Berry curvature, which is continuous, gauge invariant and reduces to the expected values at $\frac{\phi}{\phi_{0}} \rightarrow 0$. At a given magnetic flux $\phi$, let $\mathcal{P}_{\mathbf{k}}=\sum_{a=1}^{N}\left|u_{\mathbf{k}}><u_{\mathbf{k}}\right|$ be the projector to the top $C=-1$ band. The $U(N)$ non-Abelian Berry curvature is defined as $\left.F_{a b}(\mathbf{k})=-2 \operatorname{NAIm}\left(<\partial^{x} u_{\mathbf{k} a}\left|1-\mathcal{P}_{\mathbf{k}}\right| \partial^{y} u_{\mathbf{k} b}\right\rangle\right)$, where $\partial^{\mu}=\frac{\partial}{\partial k^{\mu}}$ and $A=A_{0} \phi / \phi_{0}$ is the area of the magnetic Brillouin zone. We note that the non-standard normalization NA is necessary for gauge-invariant quantities to be continuous functions of magnetic field. A semi-analytic formula for $F$, which is both numerically stable and accounts for the intrinsic geometry, is derived in the Supplementary Information. To evaluate expectation values of the Berry curvature distribution, we define the trace operator $\operatorname{tr}[O]=(\mathrm{NA})^{-1} \sum_{b=1}^{N} \int \mathrm{d}^{2} \mathbf{k} O_{b b}(\mathbf{k})$ so that $\operatorname{tr}[\mathrm{Id}]=1$, where Id is the identity operator. The Chern number $C=\operatorname{tr}[F / 2 \pi]$ is the mean of the distribution, up to $2 \pi$. The standard deviation of the Berry curvature is then defined as $\sigma(F)=\sqrt{\operatorname{tr}\left[\left(\frac{F}{2 \pi}-C\right)^{2}\right]}$.

\section{Data availability}

All data that support the plots within this paper and other findings of this study are available from the corresponding authors upon reasonable request. Source data are provided with this paper. 


\section{Code availability}

The code that supports the findings of this study is available from the corresponding authors upon reasonable request.

37. Kim, K. et al. van der Waals heterostructures with high accuracy rotational alignment. Nano Lett. 16, 1989-1995 (2016).

38. Cao, Y. et al. Superlattice-induced insulating states and valley-protected orbits in twisted bilayer graphene. Phys. Rev. Lett. 117, 116804 (2016).

39. Martin, J., Feldman, B. E., Weitz, R. T., Allen, M. T. \& Yacoby, A. Local compressibility measurements of correlated states in suspended bilayer graphene. Phys. Rev. Lett. 105 256806 (2010).

40. Bistritzer, R. \& MacDonald, A. H. Moiré butterflies in twisted bilayer graphene. Phys. Rev. B 84, 035440 (2011).

41. Hejazi, K., Liu, C. \& Balents, L. Landau levels in twisted bilayer graphene and semiclassical orbits. Phys. Rev. B 100, 035115 (2019).

42. Zhang, Y.-H., Po, H. C. \& Senthil, T. Landau level degeneracy in twisted bilayer graphene: role of symmetry breaking. Phys. Rev. B 100, 125104 (2019).

Acknowledgements We acknowledge discussions with A. Stern, Y. Sheffer and C. Repellin. This work was primarily supported by the US Department of Energy, Basic Energy Sciences Office, Division of Materials Sciences and Engineering under award DE-SC0001819 and the Gordon and Betty Moore Foundation through grant GBMF9468 to A.Y. Fabrication of samples was supported by the US Department of Energy, Basic Energy Sciences Office, Division of Materials Sciences and Engineering under award DE-SC0019300 and ARO grant number W911NF-14-1-0247. Help with transport measurements and data analysis were supported by the National Science Foundation (DMR-1809802), and the STC Center for Integrated Quantum Materials (NSF grant number DMR-1231319) (Y.C.). P.J.-H. acknowledges support from the Gordon and Betty Moore Foundation's EPiQS Initiative through grant GBMF9643. A.T.P. and P.L. acknowledge support from the Department of Defense through the National Defense Science and Engineering Graduate Fellowship Program. Y.X. and S.C. acknowledge partial support from the Harvard Quantum Initiative in Science and Engineering. A.T.P., Y.X. and A.Y. acknowledge support from the Harvard Quantum Initiative Seed Fund. A.V. was supported by a Simons Investigator award and by the Simons Collaboration on Ultra-Quantum Matter, which is a grant from the Simons Foundation (651440, A.V.). E.K. was supported by a Simons Investigator Fellowship, by NSF-DMR 1411343, and by the German National Academy of Sciences Leopoldina through a Leopoldina fellowship (grant LPDS 2018-02). P.R.F. acknowledges support from the National Science Foundation Graduate Research Fellowship under grant number DGE 1745303. This research is funded in part by the Gordon and Betty Moore Foundation's EPiQS Initiative, Grant GBMF8683 to D.E.P. K.W. and T.T. acknowledge support from the Elemental Strategy Initiative conducted by the MEXT, Japan, grant number JPMXP0112101001, and JSPS KAKENHI grant number JP2OH00354. This work was performed, in part, at the Center for Nanoscale Systems, a member of the National Nanotechnology Infrastructure Network, which is supported by the NSF under award no. ECS-0335765. The Center for Nanoscale Systems is part of Harvard University.

Author contributions Y.X., A.T.P., J.M.P., P.J.-H. and A.Y. conceived and designed the experiment. A.T.P. and Y.X. performed the scanning SET experiment and analysed the data with input from A.Y. J.M.P., Y.C. and P.J.-H. designed and provided the samples and contributed to the analysis of the results. D.E.P., E.K., P.L. and A.V. performed the theoretical analysis. K.W. and T.T. provided hBN crystals. All authors participated in discussions and in writing of the manuscript.

Competing interests The authors declare no competing interests.

\section{Additional information}

Supplementary information The online version contains supplementary material available at https://doi.org/10.1038/s41586-021-04002-3.

Correspondence and requests for materials should be addressed to Yonglong Xie,

Pablo Jarillo-Herrero or Amir Yacoby.

Reprints and permissions information is available at http://www.nature.com/reprints. 


\section{Article}
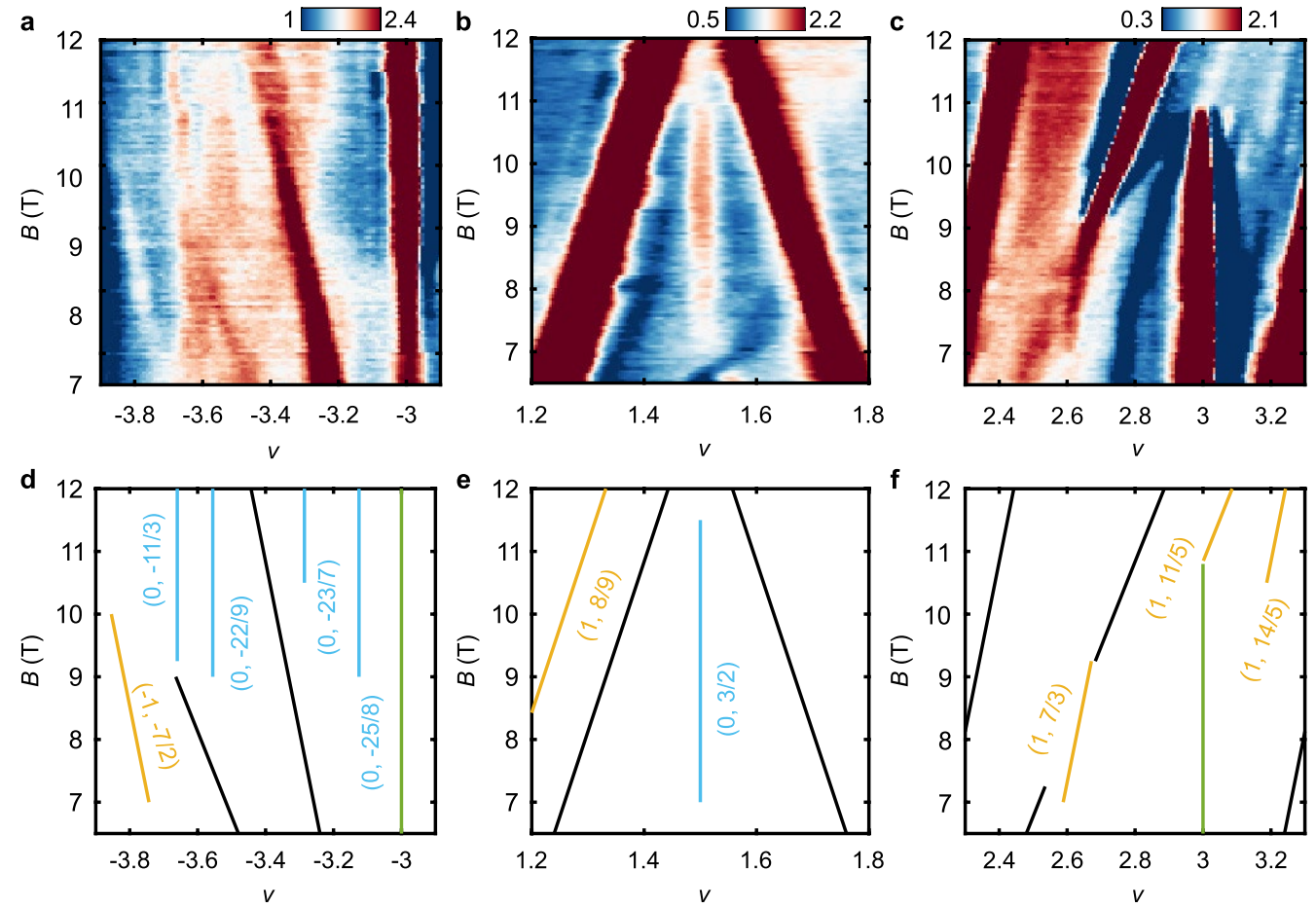

Extended data Fig. 1 | Additional CDW and SBCI states at higher magnetic field. a-d, Measurements of $\mathrm{d} \mu / \mathrm{d} n\left(\times 10^{-11} \mathrm{mV} \mathrm{cm}^{-2}\right)$ in various density ranges between 6.5 and $12 \mathrm{~T}$ showing additional CDWs and SBCIs. $\mathbf{e}-\mathbf{g}$, Schematic
Wannier diagrams corresponding to the states observed in a-d colored according to the classification used in Fig. 1b. Light blue and yellow lines denote the CDWs and SBCIs, respectively. 

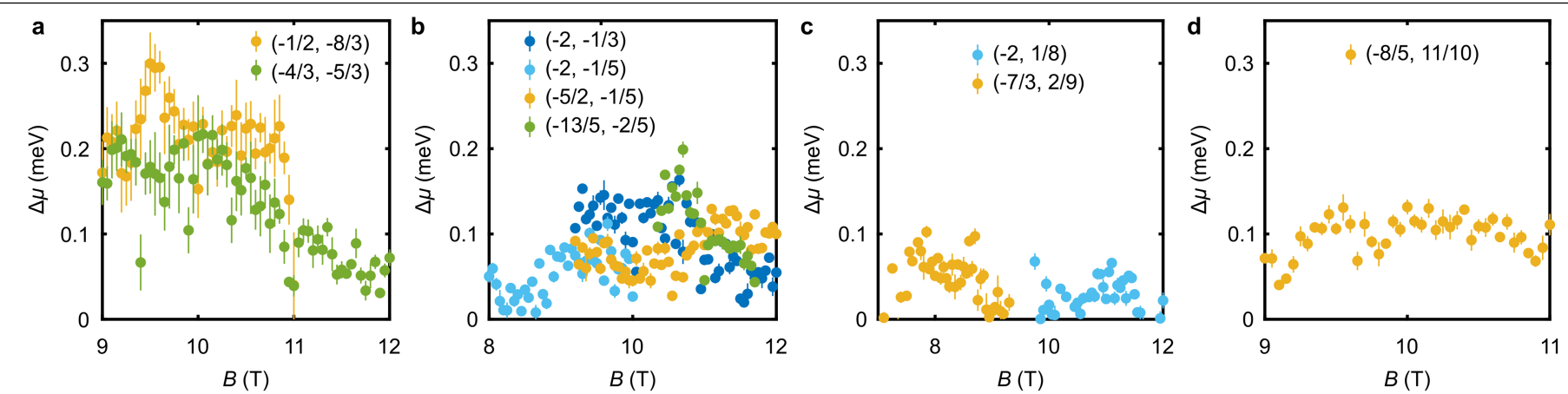

Extended Data Fig. 2 | Energy gaps of additional FCI and SBCI states. a-d, Chemical potential steps $\Delta \mu$ of the $\mathrm{FCI}$ (yellow and green circles) and SBCI (light and dark blue circles) states shown in Fig. 4. 


\section{Article}

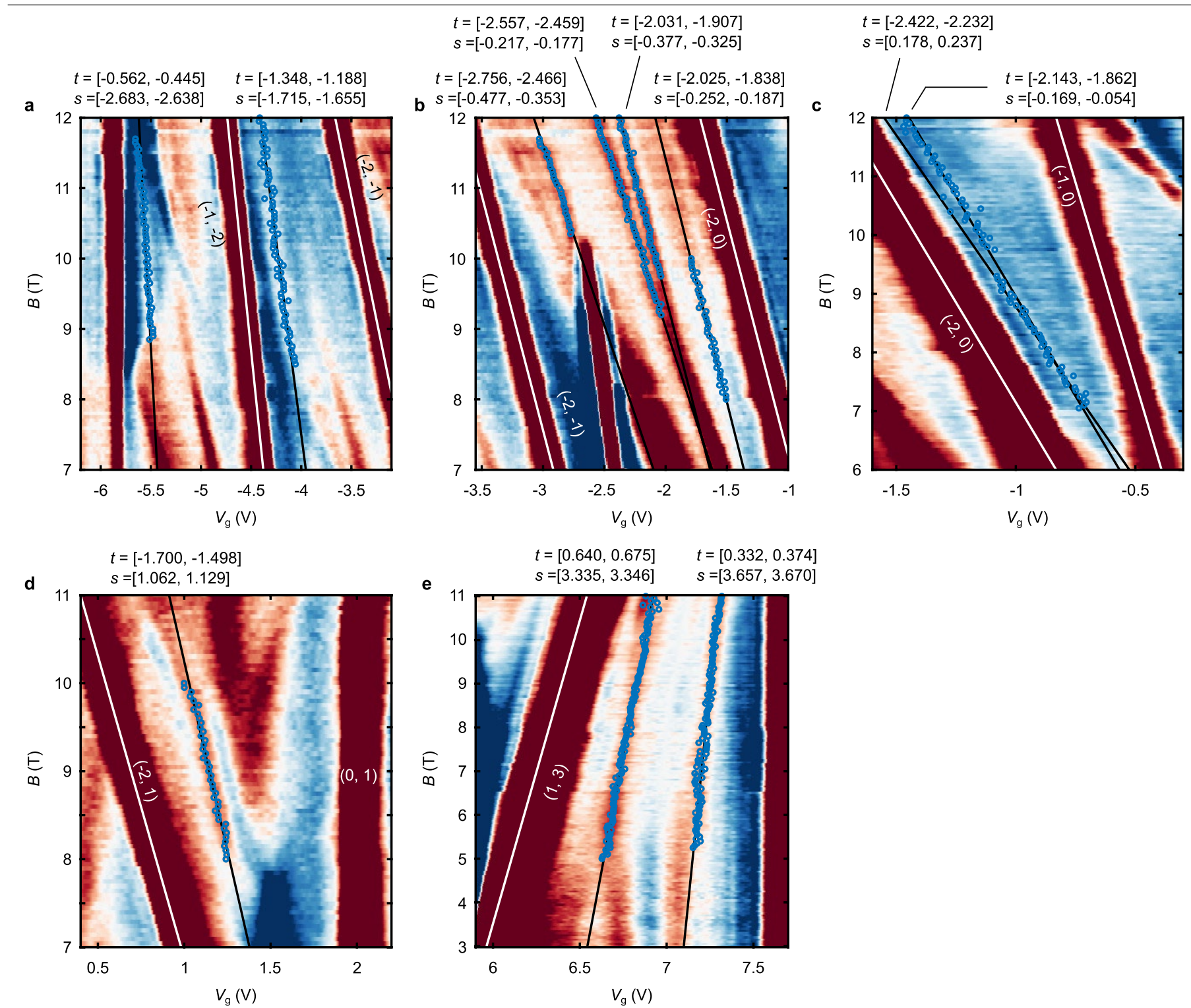

Extended Data Fig. 3 | Fits to $(t, s)$ for FCIs and SBCIs. a-e, Incompressible peak locations (blue circles) associated with $\mathrm{FCI}$ and SBCI states. Black lines mark the results of linear fits. The fitted slope of nearby Chern insulators were used to convert the parameters to $(t, s)$, the values of which are shown in the brackets with $95 \%$ confidence intervals. 


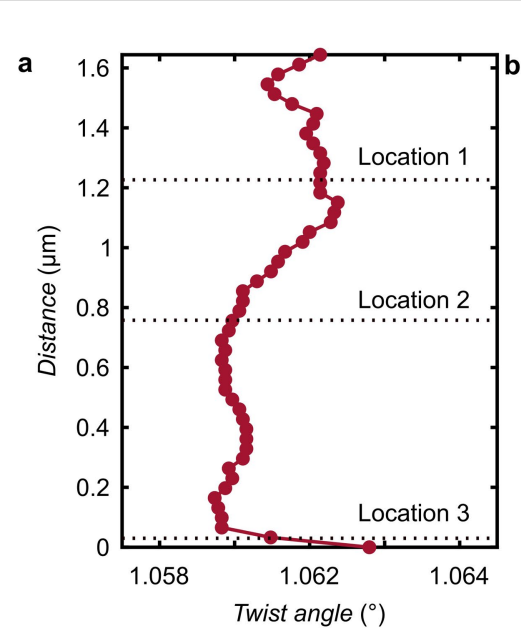

$\mathrm{d} \mu / \mathrm{d} n\left(10^{-11} \mathrm{mV} \mathrm{cm}^{2}\right) \quad-0.4 \square 0.2$

\section{$\downarrow$}

$\downarrow$

C

Location 1
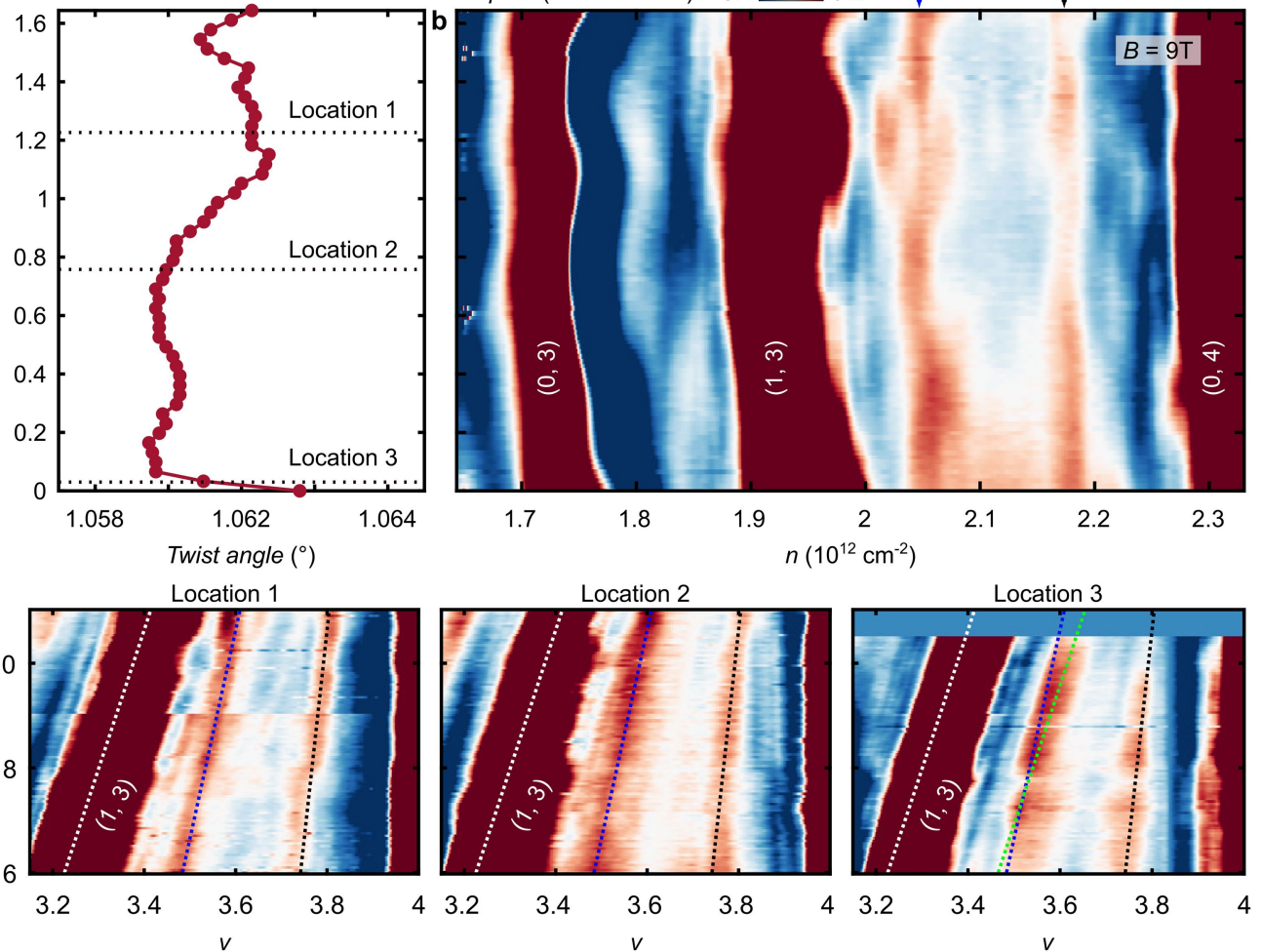

Extended Data Fig. 4 | Effect of twist angle inhomogeneity on FCIs. a, Local twist angle variation over a distance of $1.6 \mu \mathrm{m}$. b. Compressibility measurements between $v=3$ and 4 measured along the same trajectory as in a at $9 \mathrm{~T}$. The blue and black arrows indicate the densities near which the $(2 / 3$,

$10 / 3)$ and $(1 / 3,11 / 3)$ states occur, respectively. c, Compressibility measurements taken at three locations indicated by the dotted lines in $\mathbf{a}$. The white, blue, black and green dotted lines mark the expected evolution of the incompressible peaks associated with $(1,3),(2 / 3,10 / 3),(1 / 3,11 / 3)$ and $(1,13 / 4)$. 


\section{Article}

a

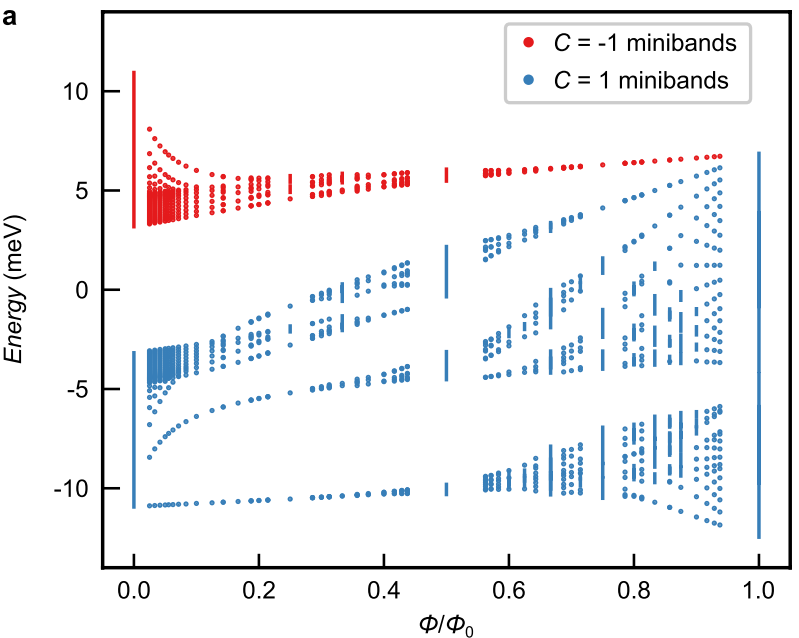

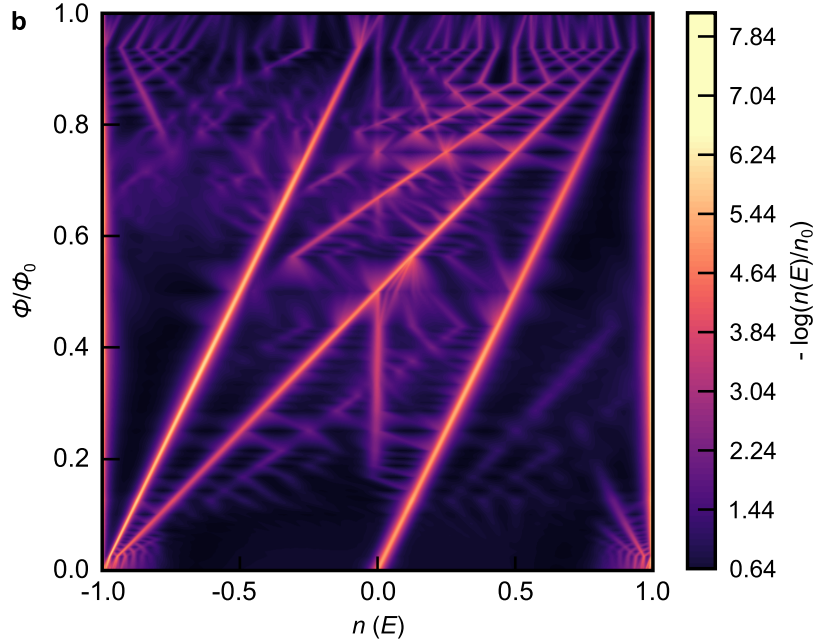

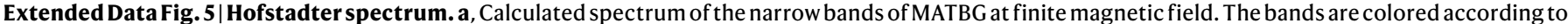
their Chern number at zero magnetic field. b, Wannier plot corresponding to the spectrum in $\mathbf{a}$. 